\title{
Les Celtes et la monnaie. Des Grecs aux surréalistes
}

Gérard Aubin

\section{OpenEdition}

Journals

Édition électronique

URL : http://journals.openedition.org/rao/6361

DOI : 10.4000/rao.6361

ISSN : 1775-3732

\section{Éditeur}

Presses universitaires de Rennes

\section{Édition imprimée}

Date de publication : 18 décembre 2020

Pagination : 246-247

ISBN : 978-2-7535-8232-3

ISSN : 0767-709X

\section{Référence électronique}

Gérard Aubin, "Les Celtes et la monnaie. Des Grecs aux surréalistes », Revue archéologique de l'Ouest [En ligne], 36 | 2020, mis en ligne le 18 décembre 2020, consulté le 03 avril 2021. URL : http:// journals.openedition.org/rao/6361; DOI : https://doi.org/10.4000/rao.6361

@ Presses universitaires de Rennes 
Chancerel A., 2013 - « Du vestige à la reconstitution du passé. Un peu d'épistémologie archéologique », Bulletin de la société d'histoire de la Guadeloupe, 165, p. 97-106.

Marquet J.-C., Verjux C., (dir.) 2012 - L'Europe, déjà, à la fin des temps préhistoriques. De grandes lames en silex dans toute l'Europe, actes de la table ronde internationale de Tours (7 septembre 2007), Joué-lès-Tours, Revue archéologique du Centre de la France, supplément 38, 242 p.

Pelegrin J., 2014 - Le phénomène pressignien : éléments d'interprétation, dans Louboutin C. et Verjux C. (dir.), Zones de production et organisation des territoires au Néolithique. Espaces exploités, occupés, parcourus, actes du $30^{\circ}$ colloque interrégional sur le Néolithique), Joué-lès-Tours, Revue archéologique du Centre de la France, supplément 51, p. 185-204.

Sheridan A., Pailler Y., 2011 - « La néolithisation de la Grande-Bretagne et de l'Irlande : plusieurs processus, plusieurs modèles et des questions à l'attention de nos collègues français ", dans Bostyn F., Martial E., Praud I. (dir.), Le Néolithique du nord de la France dans son contexte européen. Habitat et économie aux IV et III millénaires avant notre ère, actes du $29^{\circ}$ colloque interrégional sur le Néolithique, Jouélès-Tours, Revue archéologique de Picardie, numéro spécial 28, p. 13-30.

Genechesi J., Pernet L. (dir.), 2017 - Les Celtes et la monnaie. Des Grecs aux surréalistes, Gollion, Infolio, 167 p. (Document du musée cantonal d'archéologie et d'histoire, Lausanne).

Même si l'ouest de la Gaule y est peu représenté, ce catalogue de l'exposition « Les Celtes et la monnaie. Des Grecs aux surréalistes » organisée en 2017 par les musées de Lausanne, fournit un intéressant bilan des recherches récentes en numismatique celtique, dressé par une trentaine de chercheurs. Il se veut aussi un hommage à Anne Geiser, directrice du musée cantonal de 1978 à 2017. L'ouvrage est organisé de manière chronologique - des origines grecques à l'avènement d'Auguste -, en cinq parties, chacune se composant d'un copieux exposé synthétique (de 10 à 15 pages) et d'excursus plus brefs (d'une à quelques pages directement identifiables par leur fond gris) sur des techniques, des découvertes ou des sites.

Dans une première partie (p. 15-33), Lionel Pernet et Patrick Pion examinent les sources textuelles et archéologiques concernant la manière dont « la monnaie vint aux Celtes ». Ils réfutent l'idée reçue - et encore souvent exposée - d'un passage obligé du troc (une fable) à une économie monétaire, qui serait motivé par des besoins accrus en moyens d'échange. En effet, les sociétés celtiques ont fort bien vécu sans pièces de monnaies pendant plusieurs siècles et n'ont pas éprouvé le besoin d'en adopter l'usage même lorsqu'elles étaient établies aux portes de colonies grecques monétarisées comme l'était Marseille. Il y avait bien d'autres moyens de commercer et d'enregistrer les dettes économiques et sociales. Les auteurs rappellent que le besoin d'un étalon garanti naît en Grèce dans des contextes de guerres et de violence, d'appel à des mercenaires qu'il faut payer en masse (avec les questions complexes d'organisation et de logistique que rappelle Pierre Ducrey en annexe). Rapidement, cette création de richesse métallique, fondée sur la guerre, le butin, l'extraction minière et l'esclavagisme, "génère des marchés dans lesquels tout se monnaie ". Le mercenariat celtique qui s'est développé dans le monde méditerranéen a favorisé la reproduction du phénomène dans le monde celte. La frappe de monnaies - dont le choix du métal (l'or) et de l'iconographie est, dans une certaine mesure, en rupture avec les modèles grecs - résulte là aussi du besoin de financer des opérations militaires, bientôt rejoint par la multiplication de dépenses ostentatoires et le souci des aristocrates d'assurer leur prestige. La date de cette "révolution " demeure encore imprécise : peut-être dès 320300 av. J.-C (p. 28, 32, 39) pour l'utilisation de monnaies grecques (les philippes qui arrivent en Gaule sont d'ailleurs peu nombreux) tandis que l'émergence progressive de monnayages régionaux s'étalerait tout au long $\mathrm{du} \mathrm{III}^{\mathrm{e}} \mathrm{s}$. Pour leur part, Sylvia Nieto-Pelletier et Julien Olivier soulignent plusieurs points : la pluralité des modèles grecs; le rôle limité du mercenariat dans la transmission de ces modèles et celui, plus important, des lignages aristocratiques.

Dans une deuxième partie (p. 43-52), Julia Genechesi et Eneko Hiriart reviennent en détail sur l'apparition de la monnaie chez les Celtes entre 300 et 150 av. J.-C., dans un territoire allant de l'Atlantique à l'arc lémanique, en mettant en évidence des variations régionales, qu'il s'agisse du choix des prototypes (Marseille, Rhodé, Emporion), du rythme et de l'intensité de la monétarisation (un phénomène d'ampleur limitée). Aux facteurs favorables déjà évoqués (mercenariat, guerres) s'ajoute l'apparition d'agglomérations ouvertes concentrant des activités artisanales et commerciales. Selon les auteurs, les premières pièces indigènes sont émises vers 250 av. J.-C. Parmi les annexes de cette partie, on notera un développement sur les sépultures à monnaies de Suisse occidentale qui sont datées par le mobilier associé de la fin de LT C2b (c. 175-125 av. J.-C.). 
La troisième partie (p. 67-81), "Les facteurs de prospérité de la Gaule indépendante ", consacrée à la période 150-60 av. J.-C. et due à Katherine Gruel et Olivier Buchsenschutz, dresse un panorama désormais classique et qui fait consensus des acquis de la recherche archéologique depuis les années 1980 : densification et monumentalisation des fermes; développement des agglomérations artisanales; multiplication et transformation des habitats fortifiés de hauteur. Ils suggèrent que les petits bronzes et les potins dépendraient des familles aristocratiques tandis que les monnayages d'or et d'argent relèveraient des cités mais avouent que "la chronologie de leur circulation ne se précise que lentement par manque de contextes datés » (p. 72). Une place particulière est accordée aux sanctuaires à monnaies. Suivent plusieurs développements documentés sur les techniques : Katherine Gruel, "du minerai à la monnaie ", centre son propos sur l'usage de la cire perdue; Bernward Ziegaus s'intéresse à la fabrication des coins monétaires; Nicolas Consiglio et Julia Genechesi traitent des modes de fabrication des bronzes coulés appelés "potins ". Dans la mesure où la datation est une préoccupation constante de notre discipline, on apprécie le fait que la monnaie soit enfin - placée dans le contexte archéologique de sa découverte. Cette démarche est indispensable à l'établissement d'un faciès monétaire (Anne Geiser, p. 94-95) dont plusieurs exemples suisses sont donnés pour la période de l'indépendance : Vufflens-la-Ville, agglomération celtique du II $^{\mathrm{e}}$ s.; Mormont; l'oppidum de Vully, Bâle, etc. (p. 96-105).

Dans une quatrième partie (p. 107-119), Stéphane Martin et Lionel Pernet s'attachent à évaluer les conséquences de la guerre des Gaules sur les frappes monétaires qui se poursuivent et se diversifient après la Conquête, nombreuses jusque vers 30 av. J.-C. avant de cesser vers 10 av. J.-C., ainsi que sur les usages et la circulation. Ils appellent également l'attention sur le comportement de l'aristocratie militaire dans l'avant-guerre et dans l'après-guerre. Les notices complémentaires concernent Vercingétorix (Sylvia Nieto), plusieurs sites marqués par des activités militaires comme celui du mont Castel à Port-en Bessin (Anthony Lefort), les monnaies mutilées (Muriel Troubady) et, enfin, l'arrivée du numéraire romain en Narbonnaise et en Lyonnaise (Michel Amandry).

Le catalogue se clôt par un chapitre (p. 137-145) consacré à l'art dans la monnaie, abordé par Raphäl Neuville sous l'angle de l'intérêt qu'y portèrent les surréalistes, notamment à l'occasion de la parution du livre d'images de Lancelot Lengyel, L'Art gaulois dans les médailles (1954). Cette résonance est devenue un fait historique et c'est une manière intéressante d'introduire les recherches iconographiques dont on connaît les difficultés. À cet égard, j'ajouterais volontiers à la bibliographie citée par le catalogue le long compte rendu critique de l'ouvrage de Lengyel rédigé par
Pierre Merlat et Jean-Baptiste Colbert de Beaulieu (Annales de Bretagne, XLI, 1954-2, p. 337-347) lesquels, tout en saluant l'entreprise de révélation au grand public d'un art particulier, exprimaient leurs réserves sur « son interprétation uniformément ésotérique des thèmes monétaires gaulois ». Deux développements seulement dans cette partie : l'un sur le bestiaire fantastique (Dominique Hollard) tente un rapprochement des images monétaires et des textes; l'autre, "Réflexions autour de la place des femmes sur les monnaies celtiques " (Barbara Hiltmann, p. 146-148) conclut à... leur absence. Pour trouver les femmes, il est plus fécond de faire retour à l'archéologie et de s'intéresser avec Philippe Curdy, Peter Jud et Gilbert Kaenel, aux sépultures des femmes de rang et au constat que sur le Plateau suisse, " les oboles en contexte funéraire sont dédiées exclusivement à des femmes! ", ce qui conduit à des spéculations sur cette pratique et sur l'ethnie des défuntes (p. 61-63).

Au terme d'une lecture minutieuse de ce recueil, je ne formule qu'un regret, celui que la question de l'origine des métaux monétaires ne soit pas traitée alors que les questions inhérentes liées au processus minier sont fondamentales pour l'archéologie : extraction, transport et stockage, influence sur l'aménagement du territoire et le peuplement voire source de conflits internes. De même, le sujet des analyses métalliques dont l'apport a contribué à renouveler la discipline n'est que trop sommairement évoqué. Mais, au total, cette solide mise au point est pleine d'intérêt pour les archéologues de la France occidentale. N'y cherchez pas l'Ouest : sauf Port-en-Bessin (Calvados) qui bénéficie de trois pages et de quatre figures (p. 125-127), nos territoires de recherche ne sont mentionnés qu'à cinq reprises entre les pages 68 et 75 (Paule, Rannée et Liffré, Les Alleuds, Moulay, Allonnes). Mais vous y trouverez amplement matière à réflexion et à comparaison, sur la fonction et les usages de la monnaie, sur les techniques de sa fabrication, et sur sa place dans le raisonnement archéologique. Vous y ferez peut-être de vraies découvertes comme ces quatre pages captivantes de Jean-Michel Servet sur les pratiques du " commerce silencieux " (p. 34-37). Vous retiendrez qu'en dépit des avancées, la monnaie celtique ne constitue pas encore un fossile directeur très précis et qu'elle demeure un mobilier à dater. Enfin, vous éprouverez du plaisir à le consulter car ce livre soigné, répondant aux exigences scientifiques (bibliographie, glossaire, précision de la référence et du lieu de conservation du mobilier présenté dans le crédit des illustrations), fournit une illustration de grande qualité, non seulement des monnaies, mais aussi du mobilier non monétaire, des vues de sites, des plans, des cartes, des reconstitutions, etc.

Gérard Aubin 